\title{
UVEÍTIS Y ARTRITIS IDIOPÁTICA JUVENIL. EPIDEMIOLOGÍA, PRONÓSTICO Y TRATAMIENTO
}

\author{
UVEITIS AND IDIOPATHIC JUVENILE ARTHRITIS IN SPAIN. \\ EPIDEMIOLOGICAL AND THERAPEUTIC ASPECTS
}

\author{
SENDAGORTA E ${ }^{1}$, PERALTA J ${ }^{2}$, ROMERO R ${ }^{1}$, GARCÍA-CONSUEGRA R ${ }^{3}$, ABELAIRAS J², \\ ORTEGA I ${ }^{1}$
}

\begin{abstract}
RESUMEN en un centro de referencia terciario en España. vedad de la misma al diagnóstico. años). nificativas con el grupo ANA-.

\footnotetext{
Recibido: 11/7/07. Aceptado: 11/3/09.

Hospital Universitario La Paz. Madrid.

1 Licenciado en Medicina. Servicio de Oftalmología.

2 Doctor en Medicina. Servicio de Oftalmología.

3 Doctor en Medicina. Servicio de Reumatología.

Correspondencia:

Elena Sendagorta Cudós

Paseo de los Parques, 6, portal 2, 1. ${ }^{\text {a }}$

28109 Alcobendas (Madrid)

España

E-mail: elenasendagorta@hotmail.com
}

Objetivo: Describir la frecuencia y características de la uveítis asociada a la artritis idiopática juvenil

Métodos: Se estudiaron 205 niños diagnosticados de artritis idiopática juvenil en los últimos 15 años, de los cuales 26 desarrollaron uveítis anterior. Se clasificó a los pacientes según su patrón de evolución, el momento de diagnóstico de uveítis y la gra-

Resultados: La prevalencia de uveítis entre los niños con artritis idiopática juvenil fue de $12,7 \%$, de las cuales el $71 \%$ eran niñas. La edad media del diagnóstico de artritis fue significativamente menor en el grupo de pacientes que desarrollaron uveítis (4

No se observaron diferencias en la incidencia de uveítis en niños/as ni en la afectación pauci/poliarticular. Se encontraron ANA+ en el 84,6\% de los pacientes con uveítis, presentando diferencias sig-

La edad media de diagnóstico de uveítis fue de 5,87 años. El 48,1\% de los pacientes desarrollaron la uveítis en los primeros doce meses del diagnóstico de artritis. Este subgrupo de pacientes presentó más complicaciones en su evolución. El 84,7\% del total

\begin{abstract}
Purpose: The purpose of this paper is to describe the frequency and characteristics of uveitis associated with juvenile idiopathic arthritis at a tertiary referral centre in Spain

Methods: Review of clinical records. 205 children diagnosed with chronic juvenile arthritis over the last 15 years, of whom 26 presented anterior uveitis. Patients were classified according to their pattern of evolution, the time the uveitis was diagnosed with respect to the onset of arthritis, and the severity of uveitis at diagnosis.

Results: The prevalence of uveitis was $12.7 \%$. Of the patients affected with uveitis $71 \%$ were girls. The average age at which the arthritis was diagnosed was significantly earlier in the group of patients who developed uveitis (4.06 years). No differences were observed in the incidence of uveitis in girls and boys, nor in pauci/polyarticular affectation. ANA positivity was found in $84.6 \%$ of the uveitisaffected patients, this being significantly different to the ANA- group. The average age at which uveitis was diagnosed is 5.87 years. $48.1 \%$ of uveitis patients develop uveitis within 12 months of the onset of arthritis. This group presented more complications during the evolution of the condition. A
\end{abstract}


de pacientes con uveítis seguidos en consulta presentaron una agudeza visual media final $>0,5$.

Conclusiones: En nuestro estudio el desarrollo de uveítis se asocia de manera significativa con la menor edad al diagnóstico de artritis y con la presencia de ANA+. Se observa una mayor prevalencia de complicaciones en los casos que presentan un menor intervalo de tiempo entre el diagnóstico de artritis y uveítis, y en aquellos que presentaron complicaciones al diagnóstico.

Palabras clave: Uveítis, artritis idiopática juvenil, glaucoma, cataratas. total of $84.7 \%$ of the uveitis-affected patients presented with a final visual acuity of $>0.5$ during the observation period.

Conclusions: In our study, the development of uveitis was significantly associated with an earlier age of diagnosis of the arthritis and with the presence of ANA positivity. No differences were observed between boys and girls, or between pauci and polyarticular groups. A greater prevalence of complications was observed in cases presenting with a shorter time interval between the diagnosis of arthritis and uveitis and in those that presented with complications on diagnosis (Arch Soc Esp Oftalmol 2009; 84: 133-138).

Key Words: uveitis, juvenile idiopathic arthritis, cataract, glaucoma.

\section{INTRODUCCIÓN}

La uveítis asociada a la artritis idiopática juvenil es la causa más frecuente de uveítis en la población pediátrica. Tugal-Tutkun (1) publicó una serie de 130 niños con uveítis, de los cuales un $41 \%$ correspondieron a este grupo, siendo además el que más complicaciones desarrolló.

La asociación entre ambas entidades fue revisada por Rosenberg (2) (1987) y Kanski (3) (1990), y más recientemente por Kotaniemi (4) (2003). La mayoría de los estudios refieren una mayor incidencia de uveítis en la forma pauciarticular, sexo femenino y presencia de ANA+. Sin embargo, en la serie de Kotaniemi, una de las más amplias, la uveítis se desarrolló con la misma frecuencia en ambos sexos y en las formas pauci y poliarticular. Chia (5) en un estudio de casos-controles encontró una mayor incidencia de uveítis severa al diagnóstico en los niños varones. Aunque ya había sido sugerido por Rosenberg, Wolf (6) en 1987 encontró correlación entre el grado de inflamación existente en la primera consulta y la severidad en la pérdida de agudeza visual final, observando tambien una peor evolución en los casos en que la uveítis precedió a la artritis. En un reciente estudio realizado por Edelsten (7) el factor pronóstico más importante fue la uveítis severa al diagnóstico. Por tanto no existe uniformidad de criterios epidemiológicos y pronósticos en la uveítis asociada a artritis idiopática juvenil. Siendo así, aun son necesarios más estudios que den respuesta a qué probabilidad tendrá un paciente con artritis idiopática juvenil de desarrollar uveítis, y cual será el pronóstico de la misma

\section{SUJETOS, MATERIAL Y MÉTODOS}

Se incluyen en este estudio 205 niños estudiados por el servicio de oftalmología y reumatología infantil en el H.U. La Paz durante los últimos 15 años.

Los datos se recogieron mediante el estudio de historias clínicas obteniéndose los siguientes datos: Sexo, grupo de afectación articular, presencia de ANA, presencia de factor reumatoide, presencia de HLA B27, fecha de nacimiento y de diagnóstico de la artritis, fecha de primera y última revisión en reumatología y oftalmología, fecha de diagnóstico de la uveítis y de las cirugías realizadas, presencia de complicaciones en la primera consulta de oftalmología, número de brotes, presencia de síntomas, uni/bilateralidad, tiempo total de afectación en meses, tipo y tiempo total de tratamiento tópico en meses, tipo, dosis y tiempo total de tratamiento sistémico en meses, efectos secundarios del tratamiento, complicaciones en la evolución, necesidad de cirugía, tipo de cirugía realizada, agudeza visual anual a todos los niños, agudeza visual mensual a los niños intervenidos quirúrgicamente el año posterior a la cirugía, presión intraocular y excavación papilar en los hipertensos oculares.

El diagnóstico de artritis idiopática juvenil se hizo según los criterios establecidos por la Interna- 
cional League Against Rheumatism (ILAR) $(8,9)$, es decir, como una artritis de causa desconocida que acontece en pacientes menores de 16 años, con una duración de al menos seis semanas.

Los niños diagnosticados de artritis idiopática juvenil fueron remitidos a la consulta de oftalmología infantil para revisión con lámpara de hendidura y detección precoz de uveítis asintomática.

El diagnóstico de uveítis se estableció mediante examen biomicroscópico ante la presencia de flare o células (Tyndall +) en cámara anterior o precipitados queráticos recientes.

Se consideró como brote de uveítis al episodio inflamatorio separado del siguiente al menos por un mes sin actividad.

Se incluye en el grupo de uveítis desarrollada «precozmente» a aquellos niños diagnosticados de uveítis en el primer año o previo al diagnóstico de artritis.

Se incluye en el grupo de uveítis «grave» al diagnostico a aquellos que presentan complicaciones de la uveítis en la primera consulta de oftalmología. Se han considerado complicaciones de la uveítis a la presencia de sinequias, cataratas, glaucoma, queratopatía en banda y edema macular quístico.

\section{Análisis estadístico}

Los datos han sido analizados por el programa estadístico SPSS 9.0. (SPSS Inc., Chicago, Illinois, USA). La descripción de los datos cualitativos se realiza en forma de frecuencias absolutas y porcentajes. Los datos cuantitativos mediante media, mediana y desviación típica según su distribución.

La comparación entre datos cualitativos se realizó mediante el test exacto de Fischer y para datos cuantitativos entre dos grupos, el test de la $\mathrm{U}$ de Mann-Whitney (prueba no paramétrica). La correlación entre datos cuantitativos y las agudezas visuales finales se analizó mediante el coeficiente de correlación de Spearman.

Todas las pruebas estadísticas se han considerado bilaterales y como valores significativos $p<0,05$.

\section{RESULTADOS}

El tiempo medio de seguimiento de los pacientes con uveítis en la consulta de oftalmología fue de 64,2 meses (rango 6-174 meses).
La edad media de diagnóstico de artritis idiopática juvenil de los 205 niños seguidos en la consulta de Reumatología fue de 5,5 años. El 71,7\% del total de la muestra fueron niñas frente a un $28,3 \%$ de niños. La distribución por grupos según la afectación articular se resume en la tabla I.

La prevalencia de uveítis entre los niños afectados de artritis idiopática juvenil fue de $12,7 \%$. La edad media de inicio de la artritis fue de 5,73 años en el grupo sin uveítis frente a 4,06 años en el grupo con uveítis, siendo esta diferencia estadísticamente significativa $(\mathrm{p}<0,05)$.

Del total de niños con uveítis un 70,4\% de los niños pertenecen al subgrupo pauciarticular frente a un $25,9 \%$ y un $3,70 \%$ de las formas poliarticular y psoriásica. Aunque ciertamente la mayoría de los pacientes afectos de uveítis pertenecen al grupo pauciarticular, debe tenerse en cuenta que éste es el grupo más numeroso. Si observamos los porcentajes de uveítis en cada subgrupo el 15,7\% de los niños del grupo pauciarticular presentaron uveítis, frente a un $17,90 \%$ de la forma poliarticular, diferencia que no es estadísticamente significativa. Se observa también que no hay ningún caso de uveítis en la forma sistémica.

En cuanto al sexo se observa que del total de pacientes vistos en la consulta de oftalmología un $85,20 \%$ son niñas frente a un $14,80 \%$ de niños, principalmente por la mayor prevalencia de artritis idiopática juvenil en niñas. Así un 7,10\% de los niños presentarán uveítis frente a un $15,90 \%$ de las niñas. Ésta diferencia no es estadísticamente significativa.

En cuanto a la presencia de ANA+ se encontró una relación significativa $(\mathrm{p}<0,001)$ siendo ANA + el $84,6 \%$ de los niños con uveítis. El 23,7\% de los niños ANA+ desarrollaron uveítis frente al 3,70\% de los ANA-. No se ha encontrado relación significativa entre la presencia de ANA y el sexo. Del total de ANA+ el 82,7\% son niñas. El porcentaje de niños con ANA+ es de un $30 \%$ y de niñas ANA+ un

Tabla I. Afectación articular según subgrupos

\begin{tabular}{lcc}
\hline & Frecuencia & Porcentaje \\
\hline Pauciarticular & 122 & 60 \\
Sistémica & 37 & 18 \\
Poliarticular & 41 & 20 \\
Otras & 4 & 2 \\
Total & 205 & 100 \\
\hline \hline
\end{tabular}


$51,7 \%$. La edad media de diagnostico de uveítis es 5,87 años. El tiempo medio de retraso en la primera consulta oftalmológica tras el diagnóstico de artritis es de 6,6 meses.

El tiempo medio entre el diagnóstico de artritis y el de uveítis fue de 21,83 meses.

Un $48,1 \%$ de los pacientes fue diagnosticado de uveítis previamente o antes de un año desde el diagnóstico de artritis. El fenómeno más observado es el diagnóstico en la primera visita oftalmológica tras ser diagnosticado de la artritis.

En cuanto al desarrollo «precoz» (primer año o previo al diagnóstico de artritis) se observa en el $75 \%$ de los niños y en un $43,5 \%$ de las niñas. Sin embargo, la presencia/ausencia de ANA no parece tener ninguna influencia en la duración de este intervalo.

El 52\% de las uveítis no presentaron complicaciones al diagnóstico frente a un $48 \%$ que presentaron sinequias, queratopatía en banda, cataratas o hipertensión ocular desde la primera consulta. El $76,9 \%$ de los niños que fueron diagnosticados el primer año o previo al diagnóstico de artritis sufrieron complicaciones frente al 50\% de los que debutaron más tarde con la uveítis. Del total de pacientes con complicaciones un 58,8\% había sido diagnosticado el primer año o previo al diagnóstico de artritis.

Aunque las diferencias no fueron estadísticamente significativas, en el grupo de niños que desarrollaron la uveítis precozmente se observó un total de recurrencias, tiempo de afectación y tiempo de tratamiento con corticoides tópicos mayor que el otro grupo.

Se observaron complicaciones en 17 niños y 27 ojos. No se encontraron diferencias significativas en la agudeza visual final en relación a la presencia de ANA, sexo o diagnóstico precoz.

La agudeza visual media en pacientes sin complicaciones fue de 0,85 en niñas y 0,718 en niños. Un total de ocho ojos presentaron una agudeza visual final menor de 0,5 frente a 46 ojos con agudeza visual final mayor de 0,5. Requieren cirugía ocho niños y once ojos. Ocho ojos cirugía catarata, dos ojos cirugía glaucoma, un ojo cirugía vitreorretiniana.

Todos los niños intervenidos pertenecían al grupo clasificado como grave al diagnóstico (con complicaciones en la primera consulta) y habían sido diagnosticados de uveítis menos de un año después del diagnóstico de artritis.

Ocho ojos fueron operados de catarata secundaria mediante lensectomía-vitrectomía con capsulo- rrexis posterior. En ningún caso se introdujo LIO en un primer tiempo.

El tiempo medio de evolución entre el inicio de la uveítis y la necesidad de cirugía de catarata fue de 54,8 meses. La edad media a la que se intervino a estos pacientes fue de 8,9 años. Se observó una mejoría media de la agudeza visual en el optotipo tras la cirugía de 4,25 líneas a los seis meses de la cirugía de catarata.

Dos casos de un total de doce ojos precisaron cirugía de glaucoma, el resto se controló adecuadamente con tratamiento tópico.

\section{DISCUSIÓN}

La frecuencia de uveítis en los niños con artritis idiopática juvenil referida en la literatura varía entre un $2-25 \%$ siendo en nuestro estudio de un $13 \%$ $(3,9)$. Clásicamente se ha descrito una mayor incidencia de uveítis en las niñas con artritis $(3,6)$ considerándose el sexo femenino un factor de riesgo (independientemente de la mayor incidencia de artritis en las niñas) para el desarrollo de uveítis. También se ha considerado que pertenecer al grupo de afectación pauciarticular aumenta el riesgo de desarrollar uveítis.

Sin embargo, Kotaniemi (4) encuentra que no hay diferencias en la incidencia de uveítis en niñas y niños, ni entre los grupos pauci y poliarticular aunque sí cuando son ANA+.

En nuestra muestra no existen diferencias significativas en la incidencia de uveítis entre niños y niñas, ni entre los distintos grupos de afectación articular (salvo en la forma sistémica) lo que viene a apoyar esta última hipótesis. Los resultados reflejan una menor edad de diagnóstico de artritis en los niños que desarrollarán uveítis, por lo que la edad es un factor a tener en cuenta para el cribado de estos niños $(3,6,10)$. La edad media de diagnóstico de artritis en los niños que desarrollarían uveítis es de 4,06 años frente a 5,73 en los que no presentaron afectación ocular $(\mathrm{p}<0,05)$. La presencia de ANA se asocia en nuestro estudio con el desarrollo de uveítis $(\mathrm{p}<0,001)$ presentando ANA+ el 84,6\% de los niños con afectación ocular. Los valores de ANA varían en la literatura entre $71-93 \%$ en el grupo con uveítis y se considera uno de los parámetros más importantes en los programas de cribado. Se ha sugerido una peor evolución de los niños con uveítis y ANA- que no se ha observado en nuestro estudio 
(11). Los títulos de ANA no se correlacionan con la severidad de la enfermedad articular ni ocular.

Por otra parte numerosas publicaciones han tratado de identificar posibles factores asociados al desarrollo de uveítis grave, es decir predictores de una peor evolución $(5,6,12,13)$. Entre ellas destacan el intervalo entre el diagnóstico de artritis y uveítis, la severidad al diagnóstico, el sexo y el aumento de $\alpha 2$ globulinas.

Edelsten (7) y Chia (5) refieren que el sexo masculino es un factor de riesgo para el desarrollo de uveítis severa y que la evolución de los niños es peor $(4,6)$.

En nuestra muestra el $75 \%$ de los niños seguidos presentaron complicaciones en su evolución. Todos desarrollaron la uveítis menos de un año después del diagnóstico de artritis y presentaban complicaciones desde la primera consulta. Uno de ellos requirió cirugía. Por tanto éste sería un factor pronóstico de complicaciones.

El 48,1\% de los pacientes fueron diagnosticados de uveítis menos de 12 meses después o incluso antes del diagnóstico de artritis (dos casos). Aquellos que desarrollaron la uveítis en menor intervalo de tiempo, desarrollaron más complicaciones, presentaron más recurrencias y requirieron más cirugías que los que desarrollaron la uveítis de forma tardía. Esto está en consonancia con la mayoría de estudios al respecto, y parece que no hay discusión respecto a la peor evolución de los niños que desarrollan la uveítis precozmente tras el diagnóstico de artritis. En el estudio realizado por Vela (14) se observó también este fenómeno presentando el doble de episodios los pacientes con uveítis desarrollada precozmente.

Del mismo modo, $48 \%$ de los niños revisados presentaban complicaciones al diagnóstico, lo que a su vez se relaciona con una peor evolución de la uveítis, más necesidad de tratamiento e intervenciones. Este dato no difiere de otras series y refleja la necesidad de mejorar los programas de cribado. Edelsten publicó un estudio en el que la severidad de la uveítis al diagnóstico resultó el predictor más significativo de peor evolución (7). Por tanto según lo anteriormente expuesto podrían ser considerados pacientes con alto riesgo de desarrollar uveítis niños/niñas ANA+ menores de cuatro años al diagnóstico de artritis, sean pauci o poliarticulares, siendo aconsejable una revisión cada tres meses con lámpara de hendidura. Una vez transcurridos dos años esta revisión se podría realizar semestralmen- te. Los niños mayores de cuatro años o ANApodrían considerarse de riesgo medio y ser revisados semestralmente mientras que en aquellos con afectación sistémica sería suficiente con una revisión anual. Debería realizarse un seguimiento exhaustivo de los casos ya diagnosticados de uveítis que presenten factores de mal pronostico: Corto intervalo desde el diagnóstico de artritis, complicaciones al diagnóstico, varón...

Estudios más amplios permitirían elaborar protocolos y concretar el perfil de paciente que podría beneficiarse de una atención oftalmológica continuada.

Respecto a las complicaciones el $48 \%$ de los niños presentaron cataratas en su evolución. Son la complicación más frecuentemente descrita, estando en relación con el proceso inflamatorio crónico y el tratamiento con corticoides. Otros autores reflejan valores poco homogéneos desde $20 \%$ hasta $84 \%$ $(7,13,15)$, pero las series más grandes reflejan valores similares al observado en nuestra consulta $(13,16)$. El porcentaje de glaucoma secundario referido en la literatura varía entre un 15 y un $45 \%$ y en la mayoría de los casos es descrita como la complicación más devastadora $(3,14,15)$. En nuestra muestra un $33 \%$ de los niños desarrollaron episodios de hipertensión ocular, que se controlaron bien con antihipertensivos tópicos.

En nuestra serie hemos encontrado que ocho de 52 ojos presentaron una agudeza visual final menor de 0,5 y seis menor de 0,4 .

Los resultados visuales tras la cirugía de catarata obtenidos en nuestra serie son buenos. Se encuentran resultados muy variables en la literatura, lo que se explica por ser series muy pequeñas (16-18). Consideramos que el tratamiento precoz e intensivo, siguiendo el modelo de tolerancia cero a la inflamación intraocular propuesta por Foster (19), es necesario para reducir complicaciones.

En cinco ojos de ocho $(62,5 \%)$ intervenidos de catarata la agudeza visual fue mayor de 0,5 . El pronóstico visual de los niños con artritis idiopática juvenil y uveítis no ha mejorado todo lo esperado en los últimos años. Las complicaciones siguen siendo muy altas pese a los nuevos tratamientos sistémicos.

Siendo una causa importante de ceguera infantil potencialmente prevenible es necesario invertir grandes esfuerzos en la detección precoz de la enfermedad, en protocolizar el tratamiento y mejorar las técnicas quirúrgicas para prevenir complicaciones. 


\section{BIBLIOGRAFÍA}

1. Tugal-Tutkun I, Havrlikova K, Power WJ, Foster CS.Changing patterns in uveitis of childhood. Ophthalmology 1996; 103: 375-383.

2. Rosenberg AM. Uveitis associated with juvenile rheumatoid arthritis. Semin Artritis Rheum1987; 16: 158-173.

3. Kanski JJ. Juvenile arthritis and uveitis. Surv Ophhtalmol 1990; 34: 253-267.

4. Kotaniemi K, Savolainen A, Karma A, Aho K. Recent advances in uveitis of juvenile idiopathic arthritis. Surv Ophtalmol 2003; 48: 489-502.

5. Chia A, Lee V, Graham EM, Edelsten C. Factors related to severe uveitis at diagnosis in children with juvenile idiopathic arthritis in a screening program. Am J Ophthalmol 2003; 135: 757-762.

6. Wolf MD, Lichter PR, Ragsdale CG. Prognostic factors in the uveitis of juvenile rheumatoid arthritis. Ophthalmology 1987; 94: 1242-1248.

7. Edelsten C, Lee V, Bentley CR, Kanski JJ, Graham EM. An evaluation of baseline risk factors predicting severity in juvenile idiopathic arthritis associated uveitis and other chronic anterior uveitis in early childhood.Br J Ophthalmol 2002; 86: 51-56.

8. Petty RE, Southwood TR, Baum J, Bhettay E, Glass DN, Manners $P$, et al. Revision of the proposed classification criteria for juvenile idiopathic arthritis: Durban, 1997. J Rheumatol 1998; 25: 1991-1994.

9. Kotaniemi K, Kaipiainen-Seppänen O, Savolainen A, Karma A. A population-based study on uveitis in juvenile rheumatoid arthritis. Clin Exp Rheumatol 1999; 17: 119-122.

10. Zulian F, Martini G, Falcini F, Gerloni V, Zannin ME, Pinello L, et al. Early predictors of severe uveitis in oligo- articular juvenile idiopathic arthritis. J Rheumatol 2002; 29: 2446-2453.

11. Chalom EC, Goldsmith D, Koehler MA, Bittar B, Rose $C D$, Ostrov BE, et al. Prevalence and outcome of uveitis in a regional cohort of patients with juvenile rheumatoid arthritis. J Rheumatol 1997; 24: 2031-2034.

12. Cabral DA, Petty RE, Malleson PN, Ensworth S, McCormick $A Q$, Shoeder ML. Visual prognosis in children with cronic anterior uveitis and arthritis. J Rheumatol 1994; 21: 2370-2375.

13. Dana MR, Merayo-Lloves J, Schaunberg DA, Foster CS. Visual outcomes prognosticators in juvenile rheumatoid arthritis-associated uveitis. Ophthalmology 1997; 104: 236-244.

14. Vela JI, Galán A, Fernández E, Romera M, Torres JJ. Uveitis anterior y artritis idiopática juvenil. Arch Soc Esp Oftalmol 2003; 78: 561-565.

15. Kotaniemi K, Kautiainen H, Karma A, Aho K. Ocurrence of uveitis in recently diagnosed juvenile chronic artritis: a prospective study. Ophthalmology 2001; 108: 2071-2075.

16. BenEzra D, Cohen E. Cataract surgery in children with chronic uveitis. Ophtalmology 2000; 107: 1255-1260.

17. Probst LE, Holland EJ. Intraocular lens implantation in patients with juvenile rheumatoid arthritis. Am J Ophthalmol 1996; 122: 161-170.

18. Lam LA, Lowder CY, Baarveldt G, Smith SD, Traboulsi EI. Surgical management of cataracts in children with juvenile rheumatoid arthritis-associated uveitis. Am J Ophthalmol 2003; 135: 772-778.

19. Foster CS, Barrett F. Cataract development and cataract surgery in patients with juvenile rheumatoid arthritisassociated iridocyclitis. Ophthalmology 1993; 100: 809817. 